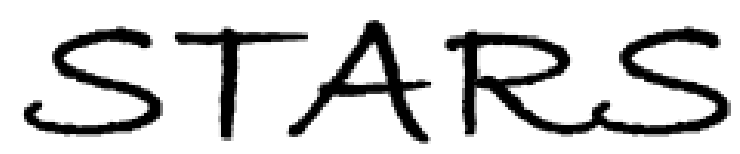

University of Central Florida

STARS

Faculty Bibliography 2000s

Faculty Bibliography

$1-1-2007$

\title{
Nuclear spectra of comet 28P Neujmin
}

Humberto Campins

University of Central Florida

Javier Licandro

Noemí Pinilla-Alonso

Kulie Ziffer

University of Central Florida

Julia De León

See next page for additional authors

Find similar works at: https://stars.library.ucf.edu/facultybib2000

University of Central Florida Libraries http://library.ucf.edu

This Article is brought to you for free and open access by the Faculty Bibliography at STARS. It has been accepted for inclusion in Faculty Bibliography 2000s by an authorized administrator of STARS. For more information, please contact STARS@ucf.edu.

\section{Recommended Citation}

Campins, Humberto; Licandro, Javier; Pinilla-Alonso, Noemí; Ziffer, Kulie; León, Julia De; Mothé-Diniz, Thais; Guerra, Juan C.; and Hergenrother, Carl, "Nuclear spectra of comet 28P Neujmin" (2007). Faculty Bibliography 2000s. 6908.

https://stars.library.ucf.edu/facultybib2000/6908

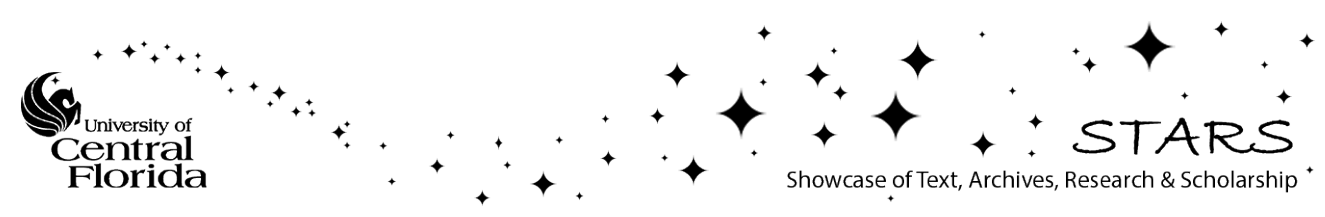


Authors

Humberto Campins, Javier Licandro, Noemí Pinilla-Alonso, Kulie Ziffer, Julia De León, Thais Mothé-Diniz, Juan C. Guerra, and Carl Hergenrother 
The Astronomical Journal, 134:1626-1633, 2007 October

(C) 2007. The American Astronomical Society. All rights reserved. Printed in U.S.A.

\title{
NUCLEAR SPECTRA OF COMET 28P NEUJMIN 1
}

\author{
Humberto Campins, ${ }^{1,2}$ Javier Licandro, ${ }^{3,4}$ Noemí Pinilla-Alonso, ${ }^{5}$ Julie Ziffer, ${ }^{1,6}$ \\ Julia de León, ${ }^{4}$ Thais Mothé-Diniz, ${ }^{7}$ Juan C. Guerra, ${ }^{3}$ and Carl Hergenrother ${ }^{2}$ \\ Received 2006 October 1; accepted 2007 May 17
}

\begin{abstract}
We present visible and near-infrared spectra of the nucleus of comet 28P/Neujmin 1, obtained in 2001, 2002, and 2003, while it had no detectable coma. The spectra show no strong features in this wavelength range, which prevented the identification of specific compounds on the surface of comet 28P. We found evidence for spectral variability, as our 2002 near-infrared spectrum has a significantly steeper slope than those obtained in 2001 and 2003. We compare the spectra of $28 \mathrm{P}$ with published spectra of other comet nuclei, with primitive asteroids and with meteorites. At nearinfrared wavelengths, all the comet nuclei show spectra with "red" slopes and the 2002 spectrum of comet $28 \mathrm{P}$ is among the reddest even when compared with Trojan asteroids. Three of the four properly observed Jupiter-family comets have significantly redder spectral slopes in the near-infrared than the one Halley-type comet in this sample. We found reasonably good matches among Trojan asteroids to the albedo and spectral shape of comet $28 \mathrm{P}$. Such similarities are consistent with an analogous formation and evolutionary environment for Trojan asteroids and Jupiter-family comets, as proposed by Morbidelli and coworkers. One CI meteorite showed a partial fit to our 2003 near-infrared spectrum of comet 28P; however, no close spectral matches to our target were found among chondritic meteorites.
\end{abstract}

Key words: comets: individual (28P) — infrared: solar system

\section{INTRODUCTION}

Jupiter-family comets are of particular interest for several reasons. These comets are the most accessible by spacecraft; in fact, four Jupiter-family comets are, or have been, targets of spacecraft missions. Jupiter-family comets are also believed to be the precursors to some Earth-crossing asteroids, and possibly the parent bodies of some meteorites (e.g., Campins \& Swindle 1998). Because most Jupiter-family comets are relatively faint they have not been studied in as much detail as Oort Cloud comets. On the other hand, Jupiter-family comets present the best opportunities to study directly the nuclear surfaces of comets. The characterization of cometary nuclear surfaces and their comparison with those of related populations such as extinct comet candidates, Centaurs, near-Earth asteroids (NEAs), trans-Neptunian objects (TNOs), and Trojan asteroids is essential to understanding the origin and evolution of small solar system bodies.

Direct observations of cometary nuclear surfaces are made difficult by gas and dust in the coma generally present when comets are close to the Sun, and by the faintness of comet nuclei at large heliocentric distances. A growing number of studies are overcoming these challenges and yielding new information on the albedos, sizes, reflectances, thermal inertias, and even composition (e.g., Abell et al. 2005; A'Hearn et al. 2005; Campins et al. 2006b; Fernández et al. 2006; Lamy et al. 2004; Licandro et al.

\footnotetext{
${ }^{1}$ Department of Physics, University of Central Florida, P.O. Box 162385 , Orlando, FL 32816-2385, USA; campins@physics.ucf.edu.

${ }^{2}$ Lunar and Planetary Laboratory, University of Arizona, Tucson, AZ 85721 , USA.

${ }^{3}$ Isaac Newton Group of Telescopes, P.O. Box 321, 38700 Santa Cruz de La Palma, La Palma, Spain.

${ }^{4}$ Instituto de Astrofísica de Canarias, C/Via Lactea s/n, 38200 La Laguna, Tenerife, Spain.

5 Fundación Galileo Galilei \& Telescopio Nazionale Galileo, P.O. Box 565, 38700 S/C de La Palma, Tenerife, Spain.

${ }^{6}$ Current address: University of Southern Maine, P.O. Box 9300, Portland, ME 04104-9300, USA.

7 Observatorio Nacional, Rio de Janeiro, Brazil.
}

2003, 2006; Sunshine et al. 2006; and references therein). The $1-2.5 \mu \mathrm{m}$ spectral region can be diagnostic of the surface compositions of comets and asteroids; however, spectroscopy of cometary nuclei in this region had not been achieved until very recently (Licandro et al. 2003; Abell et al. 2005; Sunshine et al. 2006; Campins et al. 2006b). We have continued our study of comet nuclei in the near-infrared, and in this work we present visible and near-infrared spectra of comet 28P Neujmin 1.

Comet 28P/Neujmin 1 is among the largest $(10 \mathrm{~km}$ effective radius) and least active of the Jupiter-family comets; it has an effective active area equivalent to only $0.1 \%$ of its surface (Campins et al. 1987; Campins \& Fernández 2000). Therefore, it offers a good opportunity to characterize the nuclear surface of a comet using Earth-based telescopes. Unlike most Jupiter-family comets, this comet has a rather stable orbit that has remained nearly constant due to avoidance of the perturbing influence of Jupiter. It has a Tisserand parameter with respect to Jupiter of 2.166. The Tisserand invariant is a constant of the motion in the restricted three-body problem (with Jupiter). This parameter is often used to differentiate dynamically between Jupiter-family comets $(2<$ $T<3$ ), most asteroids $(T>3)$, and Halley-type comets $(T<2)$ (e.g., Carusi et al. 1987; Levison 1996). The brightness of this comet along its orbit behaves essentially like that expected for an asteroid (Marsden 1974; Tancredi et al. 2000; Ferrin 2005); however, near perihelion weak activity has been observed. In 1984 August, at $1.7 \mathrm{AU}$ from the Sun and approximately 2 months before perihelion there was no evidence for a dust coma but gaseous emissions from $\mathrm{OH}, \mathrm{CN}, \mathrm{C}_{2}$, and $\mathrm{C}_{3}$ were detected (Campins et al. 1987). By 1984 December, at 1.8 AU from the Sun, a clearly detectable dust coma was observed (Birkett et al. 1987). Delahodde et al. (2001) used extensive observations at a large range of heliocentric distances and phase angles to refine the rotation period $(12.75 \pm 0.03 \mathrm{hr})$ and determine a phase function.

\section{OBSERVATIONS}

We obtained near-infrared $(1-2.5 \mu \mathrm{m})$ and visible spectra of comet 28P. The observing geometry for these dates and the 
TABLE 1

ObServing Geometry for 28P/Neujmin 1

\begin{tabular}{|c|c|c|c|c|c|c|}
\hline UT Date & $V$ Magnitude & $\begin{array}{c}R \\
(\mathrm{AU})\end{array}$ & $\begin{array}{c}\Delta \\
(\mathrm{AU})\end{array}$ & $\begin{array}{l}\text { Phase Angle } \\
\text { (deg) }\end{array}$ & Air Mass & Instrument/Telescope \\
\hline 2001 Apr $11.086 \ldots \ldots \ldots \ldots . . . .$. & 19 & 5.73 & 4.74 & 1.7 & 1.52 & DOLORES/TNG (visible) \\
\hline 2001 Jun $02.008 \ldots \ldots \ldots \ldots . .$. & 19 & 5.41 & 4.80 & 9.1 & 1.42 & NICS/TNG (near-infrared) \\
\hline 2002 Apr $26.163 \ldots \ldots \ldots \ldots . . .$. & 16 & 3.08 & 2.20 & 10.6 & 2.9 & NICS/TNG (near-infrared) \\
\hline 2003 Aug $25.225 \ldots \ldots \ldots \ldots . . .$. & 17 & 3.05 & 3.36 & 17.3 & 1.39 & NICS/TNG (near-infrared) \\
\hline 2003 Nov $15.151 \ldots \ldots \ldots \ldots$ & 17 & 3.69 & 2.91 & 10.8 & 1.05 & ISIS/WHT (visible) \\
\hline
\end{tabular}

instruments used are presented in Table 1 . Table 2 lists the details on the standard stars.

\subsection{Near-Infrared Spectra}

We obtained three low-resolution near-infrared spectra of comet 28P in 2001 June, 2002 April, and 2003 August (Table 1). We used the $3.56 \mathrm{~m}$ Telescopio Nazionale Galileo (TNG), with the Near-Infrared Camera Spectrometer (NICS; see Baffa et al. 2001). All three nights were photometric and had stable seeing conditions. Among the observing modes offered by the NICS instrument is a high-throughput, low-resolution spectroscopic mode with a prism disperser (Oliva 2000; Rayner et al. 2003). This mode yields a complete $0.8-2.4 \mu \mathrm{m}$ spectrum in a single exposure. A $1.5^{\prime \prime}$ width slit was used, corresponding to a spectral resolving power of 34 and quasi-constant along the spectrum. The low resolution, together with the high efficiency of the Amici prism (about $90 \%$ in this spectral range), allowed us to obtain spectra of faint objects like this comet nucleus, and with the advantage of having the whole spectral range measured simultaneously.

The object was identified at the predicted position and with the expected motion and brightness. The guider images did not show any evidence of coma activity at the time. We were unable to obtain imaging frames for quantitative limits on possible coma contribution to the flux. The slit was oriented in the parallactic angle (the position angle for which the slit is perpendicular to the horizon) to avoid problems with differential atmospheric refraction, and the tracking was at the comet's motion. The acquisition consisted of a series of images in one position (position $A$ ) of the slit and then offsetting the telescope by $10^{\prime \prime}$ in the direction of the slit (position $B$ ). This process was repeated and a number of $A B B A$ cycles were acquired. The total exposure times for each of the three dates were 3240, 4080, and $720 \mathrm{~s}$.

The reduction and calibration of all the near-infrared spectra was done as described in Licandro et al. (2006). Flat-field images and wavelength calibrations were obtained periodically through-

TABLE 2

Solar Analogs for 28P/Neujmin 1

\begin{tabular}{|c|c|c|c|}
\hline UT Date & Star & Air Mass & $V$ Magnitude \\
\hline 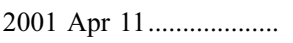 & HD 30246 & 1.71 & 8.3 \\
\hline 2001 Jun 2 .......................... & P117D & 1.06 and 1.33 & 13.5 \\
\hline 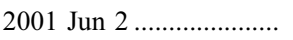 & P330E & 1.27 & 13.0 \\
\hline 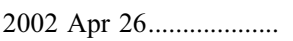 & Land $102-1081$ & 1.17 & 9.9 \\
\hline 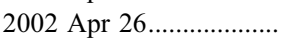 & Land $110-361$ & 1.59 & 12.4 \\
\hline 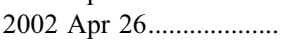 & Land $112-1333$ & 1.62 & 10.0 \\
\hline 2003 Aug $25 \ldots \ldots \ldots$ & Land $107-998$ & 1.26 & 10.4 \\
\hline 2003 Aug $25 \ldots \ldots \ldots \ldots \ldots \ldots$ & Land $112-1333$ & 1.14 & 10.0 \\
\hline 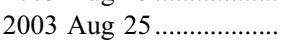 & Land $115-271$ & 1.48 & 9.7 \\
\hline 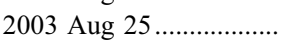 & Land 93-101 & 1.19 & 9.7 \\
\hline 2003 Nov $15 \ldots \ldots \ldots \ldots \ldots \ldots$ & Land 98-978 & 1.15 & 10.5 \\
\hline
\end{tabular}

out the observations. To correct for telluric absorption and to obtain the relative reflectance, the standard stars P117D and P330E (Colina \& Bohlin 1997) and Landolt SA 93-101, SA 102-1081, SA 107-998, SA 110-361, SA 112-1333, and SA 115-271 (Landolt 1992) were observed. These stars have colors very similar to those of the Sun (for example, the $V-I$ colors of these stars are uniform to within 2\%; Landolt 1992) and were observed during the same nights and at a similar air mass as the comet (Table 2). The Landolt stars and the solar analog P330E (Colina \& Bohlin 1997) showed similar spectra in the near-infrared region; therefore, we used the Landolt stars as local solar analogs. The flat-fielded and wavelength-calibrated spectral frames were shifted to align and stacked to provide combined frames from which the spectra were extracted. In order to obtain the relative reflectances for all three dates, plotted in Figure 1, the near-infrared spectra of the comet were divided by the mean spectrum of the solar analog stars and normalized at $1.6 \mu \mathrm{m}$. The 2001 and 2003 spectra were normalized to unity at $1.6 \mu \mathrm{m}$, while the 2002 spectrum was offset and normalized to a reflectance of 2.0 at $1.6 \mu \mathrm{m}$ for clarity (this is a vertical offset not a multiplicative one, i.e., this shift does not change the slope). The random errors for these spectra are indicated by the point-to-point variability in each spectrum and for clarity we do not show the error bars in Figure 1. Telluric conditions can vary between the comet spectra and the standard star spectra, introducing false spectral features, particularly in the two deepest bands near $1.35-1.45 \mu \mathrm{m}$ and $1.80-2.0 \mu \mathrm{m}$. The spectral points in these two telluric bands are not shown in Figure 1 and are not used in the analysis of the spectra. In the near-infrared, we see no evidence for spectral structure greater than the uncertainties. In

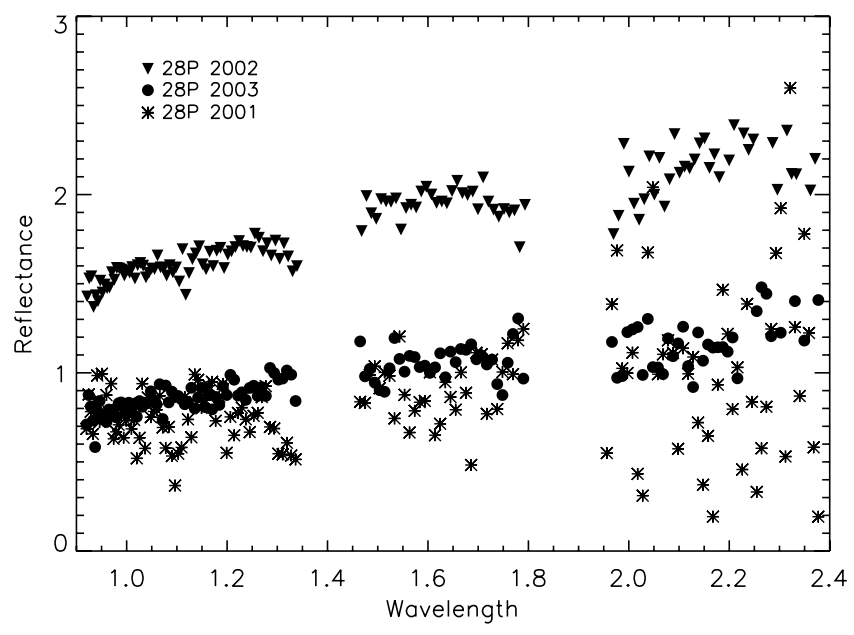

Fig. 1.-Three near-infrared reflectance spectra of comet 28P obtained in 2001 (asterisks), 2002 (triangles), and 2003 (circles), normalized at $1.60 \mu \mathrm{m}$ to 1.0 (2001 and 2003) and to 2.0 (2002); this is a vertical offset, not a multiplicative one, i.e., this shift does not change the slope. The random errors for these spectra are indicated by the point-to-point variability in each spectrum. 
TABLE 3

Spectral Slopes for 28P and Other Comet Nuclei

\begin{tabular}{|c|c|c|c|}
\hline Comet & $\begin{array}{c}\text { Visible Spectral Slope } S^{\prime} \\
\text { Normalized at } 0.55 \mu \mathrm{m} \\
\quad(\% \text { per } 1000 \AA)\end{array}$ & $\begin{array}{l}\text { NIR Spectral Slope } S^{\prime} \\
\text { Normalized at } 1.6 \mu \mathrm{m} \\
\quad(\% \text { per } 1000 \AA)\end{array}$ & $T_{\mathrm{J}}^{\mathrm{a}}$ \\
\hline 28P/Neujmin 1 ............................ & $10.3 \pm 3$ and $13.3 \pm 2$ & $2.9 \pm 0.4$ and $5.3 \pm 0.3$ & 2.166 \\
\hline 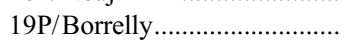 & (Not observed) & $7.7 \pm 0.8^{\mathrm{b}}$ & 2.568 \\
\hline 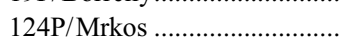 & (Not observed) & $3 \pm 1.0^{\mathrm{c}}$ & 2.752 \\
\hline 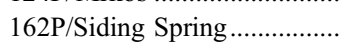 & $9.2 \pm 1.0$ & $3.6 \pm 0.3^{\mathrm{d}}$ & 2.792 \\
\hline $\mathrm{C} / 2001 \mathrm{OG}_{108} \ldots \ldots \ldots \ldots \ldots$ & $9.3 \pm 2^{\mathrm{e}}$ & $2.1 \pm 0.2^{\mathrm{f}}$ & 0.599 \\
\hline
\end{tabular}

\footnotetext{
a $T_{\mathrm{J}}$ is the Tisserand parameter with respect to Jupiter.

b Estimated from the plots in Soderblom et al. (2004).

c From Licandro et al. (2003).

d From Campins et al. (2006b)

e Calculated from the $V-R$ in Abell et al. (2005)

${ }^{\mathrm{f}}$ Estimated from the plot in Abell et al. (2005).
}

previous publications we have also normalized our near-infrared spectra at $1.6 \mu \mathrm{m}$, and in order to properly compare the slopes of our near-infrared spectra of $28 \mathrm{P}$ with other objects, the discussion below also uses a normalization to unity reflectance at $1.6 \mu \mathrm{m}$.

Two of the near-infrared spectra in Figure 1, those obtained in 2001 and 2003, are identical within the uncertainties (because of this similarity and the higher S/N, we use only the 2003 spectrum) in comparison with the spectra of related objects and in modeling (§3). The 2002 spectrum has a significantly steeper slope. Note that the continuum slope of a spectrum can be parameterized using the normalized reflectivity gradient, which is usually denoted with $S^{\prime}$ (in \%/1000 $\AA$ ), and defined as $S^{\prime}=d S / d \lambda / S^{\prime \prime}$ (e.g., Jewitt 2002). Here $S$ is the reflectivity (object flux density divided by the flux density of the Sun at the same wavelength) and $S^{\prime \prime}$ is the mean value of the reflectivity in the wavelength range over which $d S / d \lambda$ is computed. The gradient $S^{\prime}$ is used to express the percentage change in the strength of the continuum per $1000 \AA$. The use of $S^{\prime}$ facilitates comparisons with the spectral slopes of other objects; however, one must keep in mind that $S^{\prime}$ only conveys the average slope of a spectrum that is not necessarily a straight line. The slopes of the spectra from 0.8 to $2.4 \mu \mathrm{m}$ and normalized at $1.6 \mu \mathrm{m}$ are $S^{\prime}=2.9 \pm 0.4$ for 2001 and 2003 , significantly flatter than the $S^{\prime}=5.3 \pm 0.3$ found for the 2002 spectrum. This slope difference does not appear to be a systematic artifact. Although slight differences in the slope ( $\sim 2 \%$ across the entire $0.8-2.4 \mu \mathrm{m}$ region, which translates to an $S^{\prime}$ uncertainty of approximately 0.13 ) can be attributed to systematic effects resulting from the centering of the object on the slit and the seeing conditions (e.g., Cushing et al. 2004), this would not account for the observed difference. We have also considered and rejected other possible systematic sources for the slope change, including slit alignment, differential extinction, and contamination from a background source. The 2002 spectrum of the comet was obtained at a high air mass (which was necessary because of its southern declination) and the standards were observed at lower air masses (Tables 1 and 2); however, this is not the source of the steeper spectral slope. The extinction coefficients throughout this wavelength range (i.e., in the $J, H$, and $K$ atmospheric windows) are very low and nearly constant with wavelength (a detailed discussion of the extinction coefficients is given in Tokunaga et al. 2002). We estimate that the uncertainty in the spectral slope due to the air-mass difference is less than 5\%. In fact, in our extensive observations with this instrument we have never observed a correlation between spectral slope and air mass among standard stars, even at large air masses. Our estimates of the uncertainty in the value of $S^{\prime}$ includes contributions from small variations in the slopes of our standard stars. Recently, Bohlin
(2007) indicated that $\mathrm{G}$ stars with matching visible spectra may have near-infrared spectra that differ by $5 \%$. However, this uncertainty is avoided in our case by having used the same standard (SA 112-1333) for the 2002 and 2003 observations. In summary, the difference in slope between the 2002 and 2003 spectra is at least 4 times greater than all the uncertainties combined.

In Table 3 the value of $S^{\prime}$ is given for $28 \mathrm{P}$ and for the other comet nuclei observed in the near-infrared. The values for 124P and 162P are from Licandro et al. (2003) and Campins et al. (2006b), respectively. For comets 19P/Borrelly and C/2001 OG108, $S^{\prime}$ was estimated from plots in Soderblom et al. (2004) and Abell et al. (2005) (OG108).

\subsection{Visible Spectra}

Our target was observed in the visible spectral range on two separate nights using two different telescopes at "El Roque de los Muchachos" Observatory on La Palma (Canary Islands, Spain). On UT 2001 April 11.086 a spectrum was obtained with DOLORES, a camera and spectrometer for the visible permanently mounted in the Nasmyth focus of the TNG, using the LR-R grism and the 1.0" wide slit. Three $1200 \mathrm{~s}$ exposure spectra were obtained with covering the $0.5-0.91 \mu \mathrm{m}$ region and shifting the object $5^{\prime \prime}$ in the slit direction to better correct the fringing; the total exposure time was 3600 s. On 2003 UT November 15.151, we observed with the $4.2 \mathrm{~m}$ William Herschel telescope (WHT) using the red arm of the ISIS spectrograph with a $3^{\prime \prime}$ wide slit, covering the $0.52-$ $0.95 \mu \mathrm{m}$ spectral range. Three spectra of $500 \mathrm{~s}$ exposure time each were obtained, shifting the object $5^{\prime \prime}$ in the slit direction between exposures to correct the fringing. The three spectra of our target obtained on each date, at different positions of the slit, were compared in order to discard any possible problems such as background objects and finally averaged. As in the near-infrared observations, the slit was oriented in the parallactic angle and the tracking was at the object's proper motion.

Spectral data reduction was done using standard IRAF package procedures. Images were overscan and bias corrected, and flatfield corrected using lamp flats. The two-dimensional spectra were extracted, the sky background was subtracted, and the resulting spectrum collapsed to one dimension. The wavelength calibration was done using the neon and argon lamps. The solar analog stars HD 30246 and Landolt 98-978 were also observed to correct for telluric absorption and to obtain the reflectance spectra of the comet, as it was done in the near-infrared. The reduction of this spectrum was done as described in de León et al. (2004). The wavelength-calibrated visible spectra of the comet were divided by the solar analogs and binned to a spectral resolution of 240 , the 


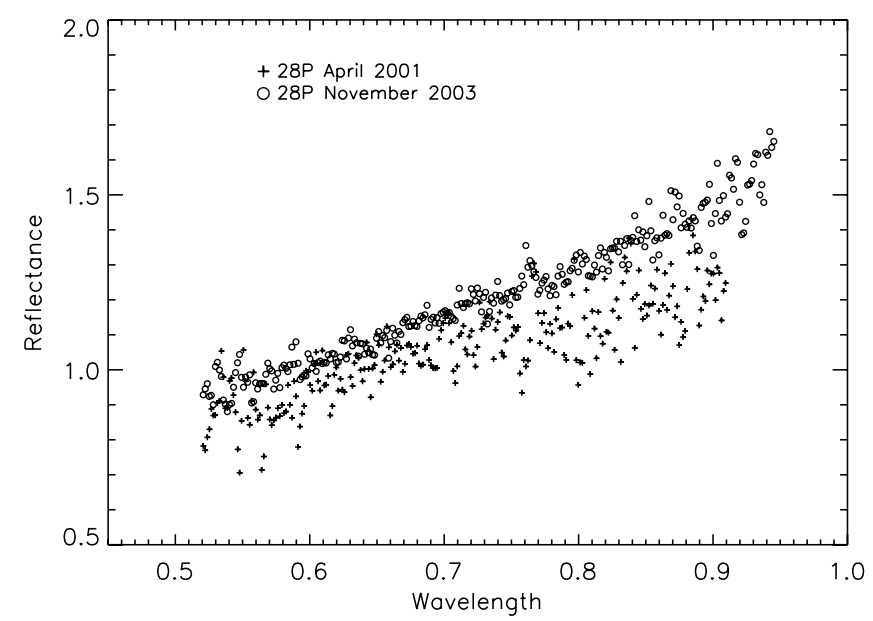

FIG. 2.-Two visible reflectance spectra of comet 28P obtained in 2001 ( plus signs) and 2003 (circles), plotted normalized to 1.0 at $0.55 \mu \mathrm{m}$. These spectra were binned to a spectral resolution of 240 . The random errors are indicated by the point-to-point variability in each spectrum.

final result is shown in Figure 2. The random errors are indicated by the point-to-point variability in the figure. At visible wavelengths it is customary to normalize spectra at $0.55 \mu \mathrm{m}$. The slopes of our two visible spectra of comet 28P, normalized at $0.55 \mu \mathrm{m}$, are $S^{\prime \prime}=10.3 \pm 3.0$ for 2001 and $S^{\prime \prime}=13.3 \pm 2.0$ for 2003 ( Table 3 ). The systematic uncertainties in the visible spectra (from the centering of the object on the slit and the seeing conditions) are such that the slope differences between our two visible spectra of comet 28P are marginal. Our two values for the visible spectral slope are essentially the same as those calculated from broadband color ratios by Delahodde et al. (2001), $S^{\prime}=9.1 \pm 1.9$, and by Jewitt \& Meech (1988), $S^{\prime}=13 \pm 4$. The overlap between 0.8 and $0.9 \mu \mathrm{m}$ in our visible and near-infrared spectra could be used to combine them; however, given that they were not obtained simultaneously and the indications of variability at both wavelengths, we chose not to combine them. Simultaneous and/or rotationally resolved spectra in future apparitions would allow a more meaningful combination of visible and near-infrared spectra.

\section{ANALYSIS AND RESULTS}

There are no strong spectral features in the wavelength range covered, and this absence of spectral structure is better defined at visible wavelengths by the lower observational uncertainties. A paucity of deep absorption features is common among low-albedo asteroids and the few comet nuclei observed in this spectral region (e.g., Abell et al. 2005; Campins et al. 2003, 2006a; Emery \& Brown 2003; Licandro et al. 2002, 2003; Fernández et al. 2004; Soderblom et al. 2004; Sunshine et al. 2006). However, as we discuss in $\S 3.6$, the overall shape of these spectra can yield useful constraints on the surface composition. In addition, the spectral variability deserves attention.

\subsection{Spectral Shape and Variability}

Visible.- In the $0.5-0.9 \mu \mathrm{m}$ region the slope and shape of our two spectra are within the range found for D-type asteroids (e.g., Jewitt 2002; see also $\S 3.4$ ). The $0.5-0.9 \mu \mathrm{m}$ slopes are also in the middle of the range of those observed in the visible for comet nuclei (e.g., Campins \& Fernández 2000). Although the slope and shape differences between our two visible spectra are marginal (Table 3 and Fig. 2), there are other indications of spectral variability in the visible and in the near-infrared. Results suggestive of a slope change in this spectral region comes from the $V, R$, and $I$ colors published by Delahodde et al. (2001). They report $V-R$ colors that range from $0.424 \pm 0.070$ to $0.574 \pm 0.043$, and $R-I$ colors from $0.400 \pm 0.05$ to $0.624 \pm 0.07$. These authors note the discrepancies, but explicitly refrain from proposing a physical explanation for the $R-I$ color changes, instead they attribute them to intrinsic problems with the $I$ filter, such as background fringing. These apparent changes in $V, R$, and $I$ colors may very well be real and simply another manifestation of the spectral variability in this comet.

Near-Infrared.-The difference between the slope of the 2002 near-infrared spectrum and those of the 2001 and 2003 spectra is clear (Table 3 and Fig. 1) and consistent with the hints of spectral variability at shorter wavelengths discussed above. This variability could be produced in three ways, rotational or seasonal (orbital aspect) variations of the surface, and a variable coma contribution; we consider these individually. (1) Rotational variability: there is spectral evidence for rotational heterogeneity on the surface of asteroids (e.g., Gaffey 1997; Rivkin et al. 2002; Campins et al. 2006a) and Centaurs (e.g., Kern et al. 2000; Licandro \& Pinilla-Alonso 2005; Merlin et al. 2005). A search for color changes at visible wavelengths through half of 28P's rotational phase (Delahodde et al. 2001) did not show detectable variations; however, as mentioned, these authors did report unexplained $V-R$ and $R-I$ color variations. (2) Orbital aspect change: if the obliquity of the rotation axis is sufficiently large, an object can show significantly different aspects to an observer at different points in the orbit. Although the orientation of the rotation axis (and hence the aspect angle) of 28P is not defined, this comet could have presented different hemispheres to the observer the times it was observed. (3) Activity: although this comet is almost never active $(\S 1)$ and there was no detectable activity at the time of the observations, activity below the spatial resolution of the images cannot be ruled out. The color of the dust coma in at least one comet, $10 \mathrm{P} /$ Tempel 2 , has exhibited visible colors less red than the nucleus (e.g., A'Hearn et al. 1989; the term "red" is commonly used to refer to spectra with increasing reflectance as a function of wavelength). However, the two least-red near-infrared spectra (in 2001 and 2003) are essentially identical, which argues against an undetected dust coma explaining identical spectra in this very low activity comet on two separate dates and at large heliocentric distances (5.7 and 3.0 AU). Interestingly, according to Beech \& Gauer (2000) the active comet with the highest likelihood of suffering impacts from meter-sized objects while in the main asteroid belt region is comet 28P. Such impacts could be responsible for sporadic activity in this comet not driven by solar radiation. However, the frequency of the spectral changes does not favor the impact option for explaining or observed variability. More specifically, according to Beech \& Gauer (2000) the time interval between possible impacts of comet 28P with meter-sized objects is of order $10^{3} \mathrm{yr}$, yet we have at least two spectral changes (one in the visible and one in the infrared) over an $18 \mathrm{yr}$ period.

\subsection{Comparison with Other Comets}

Of the comets in Table 3, visible spectra are available only for $28 \mathrm{P}$ and 162P. The two visible spectra of 28P and that of 162P are well characterized by their values of $S^{\prime}$. Hence, we chose not to make a graphical comparison of these three visible spectra. On the other hand, the near-infrared spectra show a greater diversity. Two of our near-infrared spectra of 28P (2002 and 2003) are plotted in Figure 3, along with the near-infrared spectra of comets 19P/Borrelly, 124P/Mrkos, 162P/Siding Spring, and $\mathrm{C} / 2001 \mathrm{OG}_{108}$ (LONEOS; Soderblom et al. 2004, Licandro et al. 2003, Campins et al. 2006b, and Abell et al. 2005, respectively). Each of these comet spectra is featureless within the noise and 


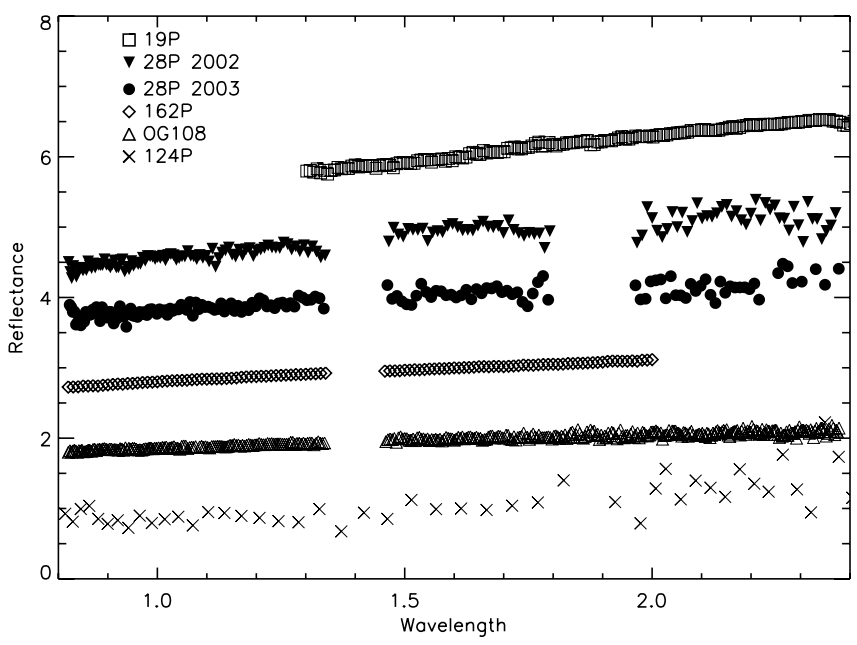

FIG. 3.-Our 2002 ( filled triangles) and 2003 (circles) near-infrared spectra of comet 28P, plotted with the spectra of comets 19P/ Borrelly (squares), 124P/Mrkos (crosses), and LONEOS OG108 (open triangles) and 162P/Siding Spring (diamonds) (Soderblom et al. 2004; Licandro et al. 2003; Abell et al. 2005; Campins et al. 2006b), all normalized to 1.0 at $1.60 \mu \mathrm{m}$ and then offset by factors of 1.0 for comparison purposes. This shift does not change the slope. The spectrum of 124P was binned to a lower spectral resolution for clarity. The point-to-point variations in the spectra are indicative of the uncertainty. The spectral slopes for these objects are listed in Table 3.

they have near-infrared slopes (in percent per $1000 \AA$, normalized at $1.60 \mu \mathrm{m}) S^{\prime}=7.7,3.0,3.6$, and 2.1, respectively (Table 3 ). The spectrum of 19P/Borrelly is very red, among the reddest observed in the solar system. We note that this spectrum was obtained by the Deep Space One mission and its reduction and calibration presented unique challenges (Soderblom et al. 2004). The steep slope $S^{\prime}=5.3$ for the 2002 spectrum of $28 \mathrm{P}$ is second only to that of $19 \mathrm{P}$ and significantly redder than those of the other four comets.

Why are the near-infrared spectra of 28P and 19P significantly redder than those of the other comets? Is this indicative of the original composition or is it evolutionary? In an attempt to resolve these questions we look at the chemical and dynamical properties of these comets. Two compositionally different groups among comets have been identified by A'Hearn et al. (1995), based on the observed ratios of the $\mathrm{CN}, \mathrm{C}_{2}$, and $\mathrm{C}_{3}$ to $\mathrm{OH}$ production rates. Depletions in $\mathrm{C}_{2}$ and $\mathrm{C}_{3}$ have been observed in some, but not all, Jupiter-family comets. Is there a manifestation of these two chemical groups in the near-infrared spectra of the comet nuclei observed? The chemical classification is available for three of the comets in Table 3. Comet 19P is classified as depleted in $\mathrm{C}_{2}$ and $\mathrm{C}_{3}$, while 28P has a "typical" composition (A'Hearn et al. 1995). C/2001 OG108 showed a "typical" composition (D. Schleicher 2006, personal communication), as expected for Oort Cloud comets (A'Hearn et al. 1995). Since the two reddest comets in this sample belong to the two chemical groups, it appears that a steep near-infrared spectrum is not associated with only one of these chemical groups (however, we remind the reader that the calibration of the spectrum of 19P is probably more uncertain than that of the other comets in Table 3). What about dynamical characteristics? We note that all but one of the comets in Table 3 are Jupiter-family comets, the exception being C/2001 OG108, which is a Halley-type comet with likely origin in the Oort cloud. In this very limited sample, three of the four Jupiter-family comets have significantly redder $S^{\prime}$ values in the near-infrared than the one Halley-type comet, and the larger uncertainty puts the value of $S^{\prime}$ for $124 \mathrm{P}$ within that for $\mathrm{C} / 2001$ OG108. We found no correlation between the Tisserand parameter $T_{\mathrm{J}}$ and $S^{\prime}$ (Table 3). A cor-

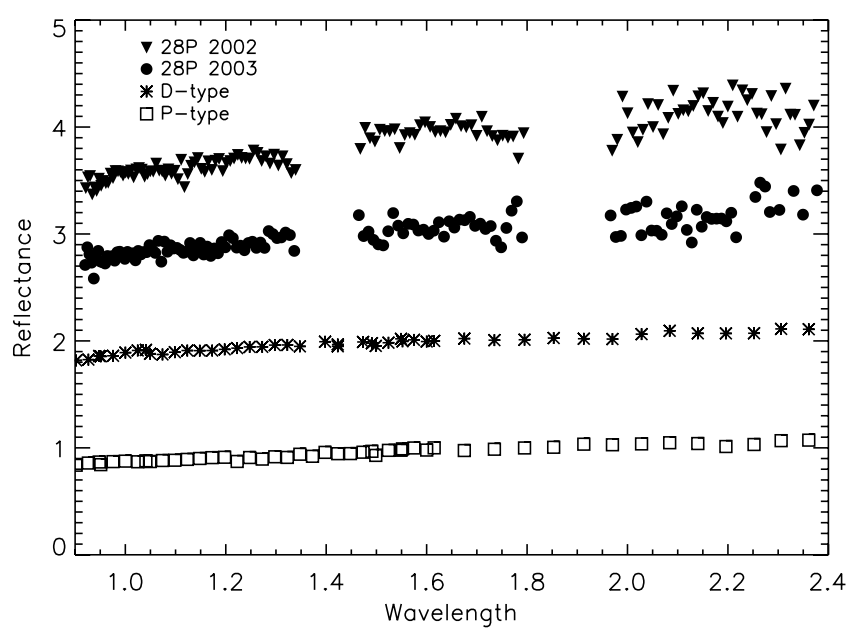

FIG. 4.-The 2002 (triangles) and 2003 (circles) near-infrared reflectance spectra of comet 28P are plotted with the average spectra of three representative P-type asteroids (squares) and three representative D-type asteroids (asterisks) (see text for references). The spectra were normalized to 1.0 at $1.60 \mu \mathrm{m}$ and offset by factors of 1.0 for clarity; this shift does not change the slope.

relation between the visible $S^{\prime}$ (normalized at $0.55 \mu \mathrm{m}$ ) and the near-infrared $S^{\prime}$ (normalized at $1.6 \mu \mathrm{m}$ ) is apparent among the three relevant objects in Table 3. We emphasize that all these considerations about comets are very preliminary, since our sample is so limited, and they must be revisited as new objects are observed.

\subsection{Comparison with Spectra of Asteroids}

Low-albedo "primitive" asteroids in the outer main belt as well as in the Trojan clouds also have red spectra with little or no structure. By primitive asteroids we mean $\mathrm{C}, \mathrm{P}$, and $\mathrm{D}$ types in the Tholen classification scheme (Tholen \& Barucci 1989); some of these primitive asteroids may be related to comets. In Figure 4 we compare our near-infrared spectra of comet $28 \mathrm{P}$ with the average spectra of three representative main-belt P-type asteroids (65 Cybele, 76 Freia, and 476 Hedwig) and three representative main-belt D-type asteroids (336 Lacadiera, 368 Haidea, and 773 Irmintraud; Zellner et al. 1985; Bell et al. 1988). Although the slopes of our two visible spectra of $28 \mathrm{P}$ are close to the average of D-type asteroids $\left(S^{\prime}=9.1 \pm 1.1\right.$; Fitzsimmons et al. 1994), in the $0.8-2.4 \mu \mathrm{m}$ region the 2002 spectrum of $28 \mathrm{P}$ is significantly redder than the $\mathrm{P}$ and $\mathrm{D}$ types plotted, which have $S^{\prime}$ values of 1.9 and $2.4 \pm 0.2$, respectively. Our other two near-infrared spectra of comet 28P (2001 and 2003) are the same, within the uncertainties, as those of the asteroids. The D-type asteroid 944 Hidalgo, which also shows spectral variability in the near-infrared (Campins et al. 2006a), is in a cometary orbit, and dynamically it is considered the most likely asteroid to be of cometary origin.

Recently, Morbidelli et al. (2005) provided dynamical arguments indicating that Trojan asteroids may have formed in more distant regions and were subsequently captured into co-orbital motion with Jupiter. In addition, escaped Trojans are considered one possible source of Jupiter-family comets (e.g., Marzari et al. 2002). Since it is well established that the visible spectra of Trojans are similar to those of inactive comet nuclei (e.g., Jewitt 2002 and references therein), we concentrate on comparisons of near-infrared spectra. We compared our near-infrared spectrum with those of 14 Trojan asteroids observed at similar wavelengths by Emery \& Brown (2003). We found reasonable matches to our 2003 spectrum 


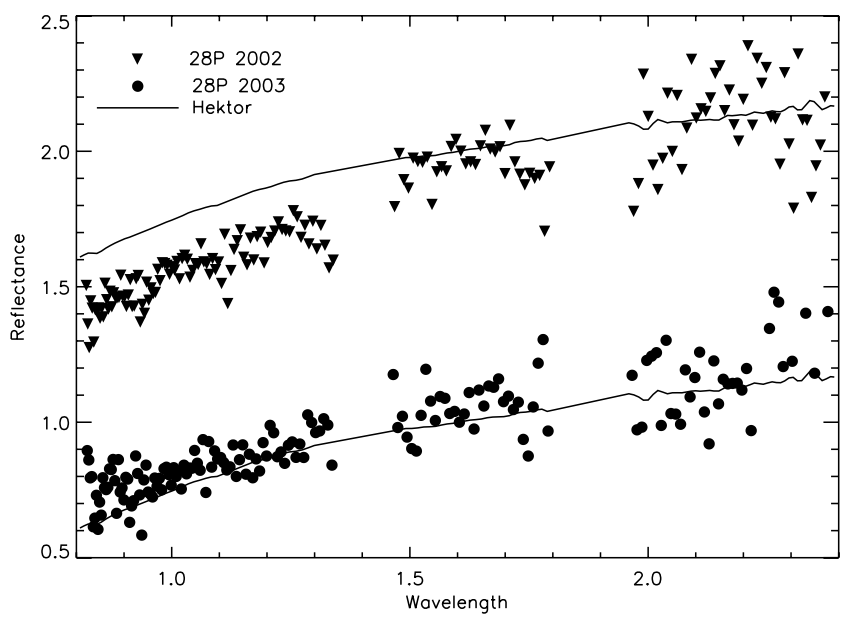

FIG.5.-The 2002 (triangles) and 2003 (circles) near-infrared spectra of comet 28P, plotted with the Trojan asteroid 624 Hektor (line) from Emery \& Brown (2003). The spectra are normalized at $1.60 \mu \mathrm{m}$ to 2.0 and 1.0 , respectively. The normalization shift does not change the slope.

among several of the Trojans. The closest Trojan asteroid spectrum to our 2002 spectrum is that of asteroid 624 Hektor (Fig. 5), which is the reddest of the Trojan asteroids observed so far at these wavelengths (Emery \& Brown 2003); note that the slopes and overall spectral shapes of these two objects are very similar. Interestingly, the $V$-band geometric albedos $\left(p_{v}\right)$ for 624 Hektor, $3 \% \pm 1 \%$ (Cruikshank 1977), and for comet 28P, $2.5 \% \pm 0.8 \%$ (Campins \& Fernández 2000), are essentially identical. The spectral and albedo similarities may be indicative of analogous compositions (§ 3.6). At the same time, the spectral diversity among Trojan asteroids is also comparable with that seen among the few comet nuclei observed so far in the near-infrared (Campins et al. 2006b). In other words, among Trojan asteroids we find reasonable matches to individual comet spectra, as well as to the spectral range observed so far among comet nuclei. Such similarities are consistent with analogous formation and evolutionary environments for both populations, as proposed by Morbidelli et al. (2005). One caveat to be considered is the size difference between the Jupiter-family comets and the Trojan asteroids used in this comparison, i.e., those for which near-infrared spectra have been obtained. The sample of Jupiter-family comets have radii of $10 \mathrm{~km}$ or less, while this sample of Trojan asteroids have radii greater than $100 \mathrm{~km}$.

\subsection{Comparison with Centaurs and Trans-Neptunian Objects}

Comets are believed to be dynamically related to Centaurs and TNOs, and near-infrared spectra of these objects are increasingly available (see review by Barucci et al. 2004 and references therein). Some of these objects have featureless spectra in the near-infrared but others do not. The presence of strong absorption bands and the range of observed spectral characteristics suggest that many of the Centaurs and TNOs have surface compositions different from those found among comet nuclei (e.g., Cruikshank et al. 1998; Jewitt 2002; Licandro et al. 2001, 2002, 2006; Barucci et al. 2004). Possible processes that would explain such compositional differences are discussed in the references cited above. Among Centaurs and TNOs there is a broad range of visible colors, broader than observed so far in comet nuclei (Jewitt 2002). However, in the near-infrared our spectra of comets $28 \mathrm{P}$ are just as red or redder than Centaur 5145 Pholus and as red or redder than most TNOs. To illustrate this, we plot in Figures 6 and 7 our 2002 near-infrared spectrum of $28 \mathrm{P}$ with those of 5145 Pholus

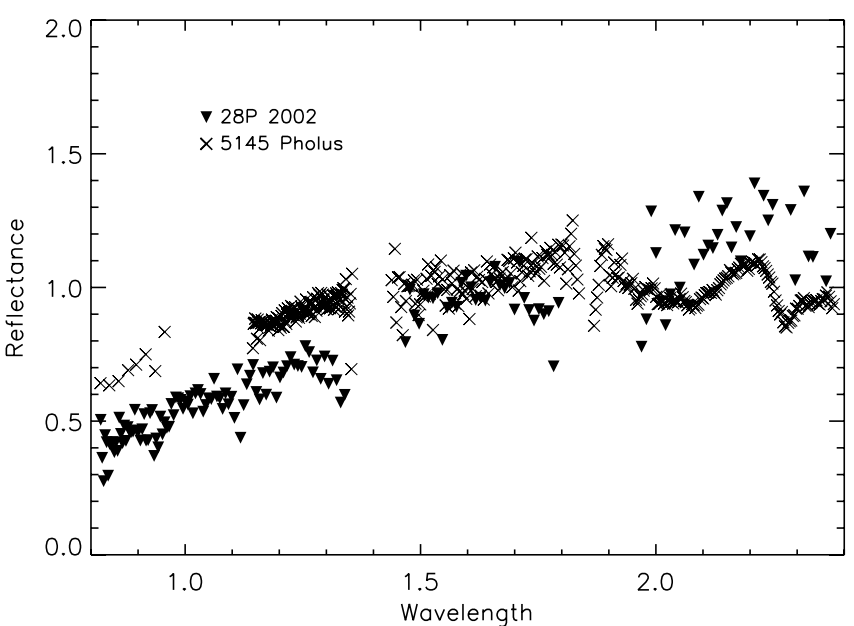

FIG. 6.-The 2002 (triangles) near-infrared spectrum of 28P, plotted with Centaur 5145 Pholus (crosses; Cruikshank et al. 1998). The spectra are normalized at $1.60 \mu \mathrm{m}$ to 1.0 .

(Cruikshank et al. 1998) and 50,000 Quaoar (Pinilla \& Licandro 2004).

\subsection{Comparison with Meteorite Spectra}

Comparison of the visible and near-infrared spectra with meteoritic samples can help constrain the composition of comets. As with comparisons between asteroids and meteorites, care must be taken since terrestrial weathering can contaminate the meteoritic samples and space weathering can influence the spectra of asteroids (e.g., Clark et al. 2002). Our approach is similar to that used to compare the spectrum of comet 162P/Siding Spring with meteorites (Campins et al. 2006b); however, in the case of comet $28 \mathrm{P}$ we limit ourselves to fitting the $0.8-2.4 \mu \mathrm{m}$ spectra. We used the Relab database (Pieters \& Hiroi 2004), which contains 802 meteorite samples to search for appropriate fits to our spectra. A first-order automatic search was performed by interpolating both the meteorite and the comet spectra to a common wavelength sampling in the spectral range observed. The dispersion between the resampled comet and meteorite spectra were then calculated for each spectrum in the database and sorted in ascending order of dispersion. A visual inspection of the spectra of the comet and meteorites according to the resulting list was

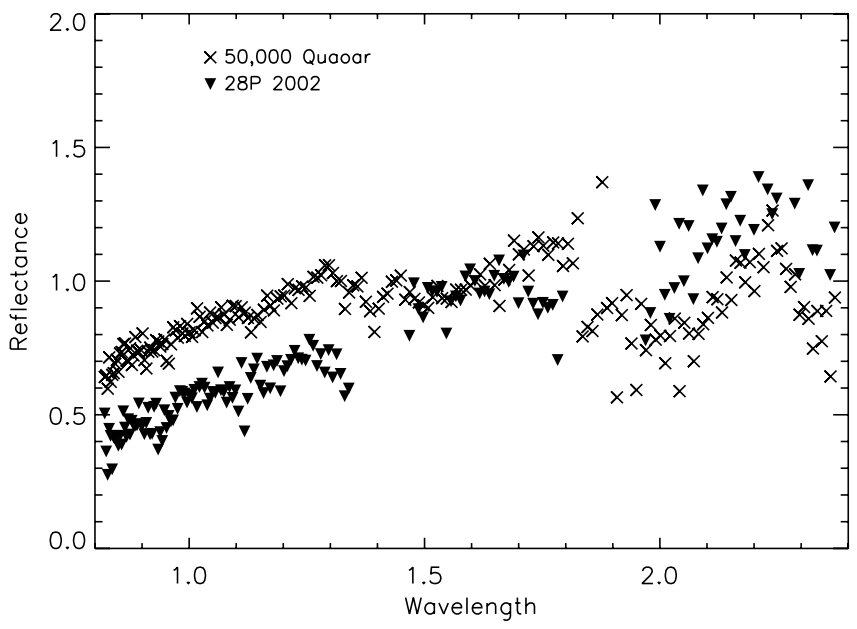

FIG. 7.-The 2002 (triangles) near-infrared spectrum of 28P, plotted with the TNO 50,000 Quaoar (crosses) (Pinilla \& Licandro 2004). The spectra are normalized at $1.60 \mu \mathrm{m}$ to 1.0 . 


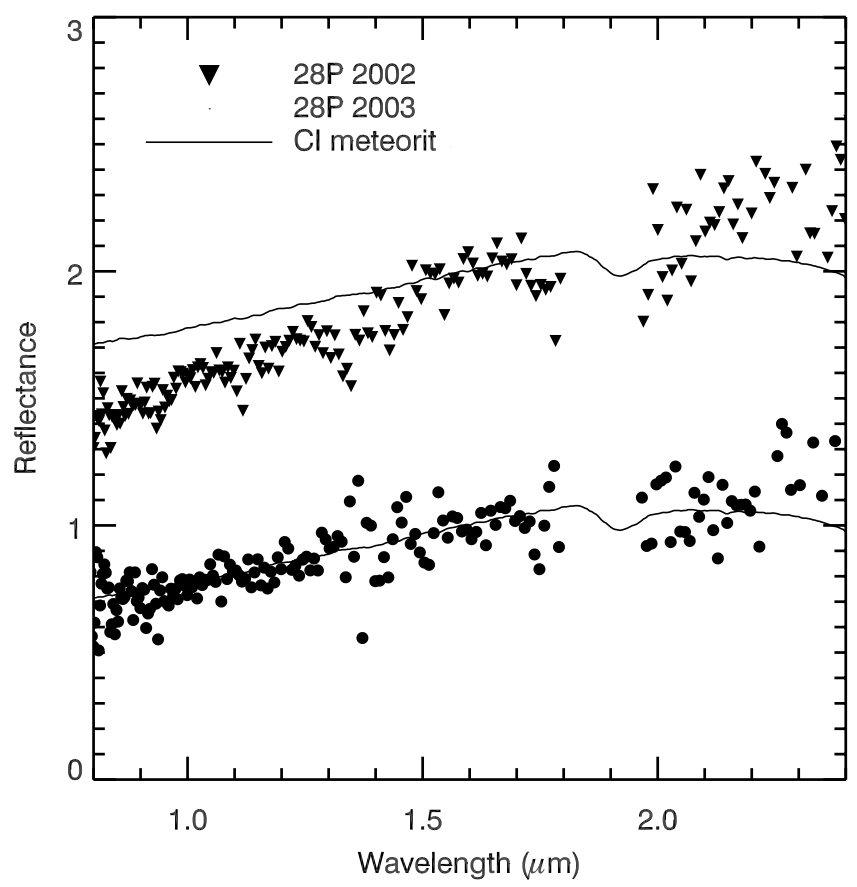

FIG. 8.-The 2002 (triangles) and 2003 (circles) near-infrared reflectance spectra of comet $28 \mathrm{P}$ normalized at $1.60 \mu \mathrm{m}$ to 1.0 (2003) and to 2.0 (2002), compared with that of a CI carbonaceous chondrite meteorite (Relab sample MR-MJG-106). This is the same meteorite that gave the closest (although not very good) fit to our visible and near-infrared spectrum of comet 162P (Campins et al. 2006b). The normalization shift does not change the slope.

then performed. The best fit to a primitive meteorite was obtained with the Alais CI carbonaceous chondrite (Relab sample MR-MJG-106), which is shown in Figure 8. As in the previous comparison figures, we do not plot the 2001 spectrum of $28 \mathrm{P}$ because it is essentially identical to the 2003 spectrum. The fit is better for the 2003 spectrum but not ideal; in contrast with the spectra of 28P, the meteorite reflectivity decreases beyond $2.2 \mu \mathrm{m}$. Although this partial fit to the 2003 spectrum is interesting, the relatively low signal-to-noise ratio of the $28 \mathrm{P}$ spectra prevent a more detailed interpretation of this similarity. We note that this same meteorite also gave the closest fit to the $0.3-$ $2.4 \mu \mathrm{m}$ spectrum of comet $162 \mathrm{P}$. However, the fit to comet $162 \mathrm{P}$ was not very good, with the most significant shape discrepancies in the ultraviolet, visible, and $1.9 \mu \mathrm{m}$ regions (Campins et al. 2006b). As more comet and meteorite spectra become available they will likely play an important role in the identification of cometary meteorites.

\subsection{Models}

Modeling of the surface spectra of atmosphereless solar system objects to infer their composition can be a powerful analytical tool and has been discussed in a number of publications (e.g., Hapke 1981; Shkuratov et al. 1999; Cruikshank et al. 1998; Emery \& Brown 2004; Clark et al. 2004). We used the scattering theory described by Shkuratov et al. (1999) for areal mixtures. Our approach is very similar to that used for modeling Trojan asteroids (Emery \& Brown 2004) and Centaurs (e.g., Cruikshank et al. 1998; Poulet et al. 2002). The main constraints are the albedo $\left(p_{v}=2.5 \% \pm 0.8 \%\right.$; Campins \& Fernández 2000) and the overall shape of the spectrum. However, the relatively large observational uncertainties in our near-infrared spectra and the absence of strong spectral features prevents the identification of specific minerals, and our resulting model compositions of comet 28P are not unique. Furthermore, our modeling approach needs to be refined. If taken literally, our models of comet 162P (Campins et al. 2006b) would suggest that the surface of that comet could be mostly carbon, with olivine, pyroxene, and organic compounds as probable components. This high carbon abundance is unlikely, since carbon-rich meteorites (with albedos as low as those of comet nuclei) have at most $3.2 \%$ by weight of carbon (Hutchison 2004, p. 29). The use in our models of carbons with albedos close to those of comet nuclei required most of the modeled surface to be carbon, and even with a very high carbon abundance $(\sim 85 \%)$ we were unable to match the observed albedo of comet $28 \mathrm{P}$. It is also important to remember that carbon is not the only way to make a surface dark; for example, various vaporization and sputtering mechanisms can cover silicates with submicroscopic iron particles, making them darker. Therefore, more rigorous modeling should include carbon species with lower albedo and other lowalbedo minerals. Given the current limitations of the models and of the observations, we refrain from further interpretation of our modeling results.

\section{CONCLUSION}

We found evidence for spectral variability in the nucleus of comet 28P: our 2002 near-infrared spectrum has a significantly steeper slope than those obtained in 2001 and 2003. Our observations provide additional evidence that bare comet nuclei have spectra with muted or absent structure, low albedos, and nearinfrared spectra with red slopes. Three of the four properly observed Jupiter-family comets have significantly redder spectral slopes in the near-infrared than the one Halley-type comet in this sample. So far, the nuclei of comets appear spectrally similar to primitive asteroids, primarily D-type asteroids. The few comet nuclei observed spectroscopically in the near-infrared exhibit considerable diversity, similar to that found among near-infrared spectra of Trojan asteroids by Emery \& Brown (2003). We found reasonably good matches among Trojan asteroids to the albedo and spectral shape of comet 28P. Such similarities are consistent with an analogous formation and evolutionary environment for Trojan asteroids and Jupiter-family comets, as proposed by Morbidelli et al. (2005). One CI meteorite showed a partial fit to our 2003 near-infrared spectrum of comet 28P; however, no close spectral matches to our target were found among chondritic meteorites.

We thank J. Emery and P. Abell for providing their original data and for helpful comments. H. C. was supported by grants from NASA's Planetary Astronomy and from the National Science Foundation. J. Z. was supported by a fellowship from the Florida Space Grant Consortium and by a grant from the National Science Foundation. T. M. D. acknowledges the support of a postdoctoral fellowship from the Brazilian government's CNPq. This paper is based on observations made with the Italian Telescopio Nazionale Galileo ( TNG) operated on the island of $\mathrm{La}$ Palma by the Centro Galileo Galilei of the CNAA (Consorzio Nazionale per l'Astronomia e l'Astrofisica) and with the William Herschel Telescope (WHT) operated by the Isaac Newton Group, both telescopes are at the Spanish Observatorio del Roque de los Muchachos of the Instituto de Astrofisica de Canarias. We are grateful to all the technical staff and telescope operators of the TNG and WHT for their assistance. We also thank the referee, M. Sitko, for very constructive comments. 
Abell, P. A., et al. 2005, Icarus, 179, 174

A'Hearn, M. F., Campins, H., Schleicher, D. G., \& Millis, R. L. 1989, ApJ, 347, 1155

A'Hearn, M. F., Millis, R. L., Schleicher, D. G., Osip, D. J., \& Birch, P. V. 1995, Icarus, 118, 223

A'Hearn, M. F., et al. 2005, Science, 310, 258

Baffa, C., et al. 2001, A\&A, 378, 722

Barucci, M. A., Doressoundiram, A., \& Cruikshank, D. P. 2004, in Comets II, ed. M. C. Festou, H. U. Keller, \& H. A. Weaver (Tucson: Univ. Arizona Press), 647

Beech, M., \& Gauer, K. 2000, Earth Moon Planets, 88, 211

Bell, J. F., Owensby, P. D., Hawke, B. R., \& Gaffey, M. J. 1988, in Lunar \& Planetary Science XIX, ed. G. Ryder \& V. L. Sharpton (Houston: LPI), 57

Birkett, C. M., Green, S. F., Zarnecki, J. C., \& Russell, K. S. 1987, MNRAS, 225,285

Bohlin, R. C. 2007, in ASP Conf. Ser. 364, The Future of Photometric, Spectrophotometric, and Polarimetric Standardization, ed. C. Sterken (San Francisco: ASP), 315

Campins, H., A’Hearn, M. F., \& McFadden, L. 1987, ApJ, 316, 847

Campins, H., \& Fernández, Y. 2000, Earth Moon Planets, 89, 117

Campins, H., Licandro, J., Fernández, Y., Hergenrother, C., Ziffer, J., Emery, J., Cruikshank, D., \& Pinilla-Alonso, N. 2006a, AAS DPS Meeting, 38, 20.04

Campins, H., Licandro, J., Guerra, J., Chamberlain, M., \& Pantin, E. 2003, BAAS, 35, 1011

Campins, H., \& Swindle, T. D. 1998, Meteoritics Planet. Sci., 33, 1201

Campins, H., Ziffer, J., Licandro, J., Pinilla-Alonso, N., Fernández, Y., de Leon, J., Mothe-Diniz, T., \& Binzel, R. P. 2006b, AJ, 132, 1346

Carusi, A., Kresak, L., Perozzi, E., \& Valsecchi, G. B. 1987, A\&A, 187, 899

Clark, B. E., Bus, S. J., Rivkin, A. S., Shepard, M. K., \& Shah, S. 2004, AJ, 128,3070

Clark, B. E., Hapke, B., Pieters, C., \& Britt, D. 2002, in Asteroids III, ed. R. Binzel et al. (Tucson: Univ. Arizona Press), 585

Colina, L., \& Bohlin, R. 1997, AJ, 113, 1138

Cruikshank, D. P. 1977, Icarus, 30, 224

Cruikshank, D. P., et al. 1998, Icarus, 135, 389

Cushing, M. C., Vacca, W. D., \& Rayner, J. T. 2004, PASP, 116, 362

Delahodde, C. E., Meech, K. J., Hainaut, O. R., \& Dotto, E. 2001, A\&A, 376, 672

De León, J., Duffard, R., Licandro, J., \& Lazzaro, D. 2004, A\&A, 422, L59

Emery, J. P., \& Brown, R. H. 2003, Icarus, 164, 104 2004, Icarus, 170, 131

Fernández, Y. R., Campins, H., Kassis, M., Hora, J. L., Hergenrother, C. W., Binzel, R. P., \& Licandro, J. 2006, AJ, 132, 1354

Fernández, Y. R., Lisse, C. M., Schleicher, D. G., Bus, S. J., Kassis, M., Hora, J. L., \& Deutsch, L. K. 2004, BAAS, 36, 1117

Ferrin, I. 2005, Icarus, 178, 493

Fitzsimmons, A., Dahlgren, M., Lagerkvist, C.-I., Magnusson, P., \& Williams, I. P. 1994, A\&A, 282, 634

Gaffey, M. J. 1997, Icarus, 127, 130

\section{EFERENCES}

Hapke, B. 1981, J. Geophys. Res., 86, 4571

Hutchison, R. 2004, Meteorites (Cambridge: Cambridge Univ. Press)

Jewitt, D. 2002, AJ, 123, 1039

Jewitt, D. C., \& Meech, K. J. 1988, ApJ, 328, 974

Kern, S. D., McCarthy, D. W., Buie, M. W., Brown, R. H., Campins, H., \& Rieke, M. 2000, ApJ, 542, L155

Lamy, P. L., Toth, I., Fernández, Y. R., \& Weaver, H. A. 2004, in Comets II, ed. M. C. Festou, H. U. Keller, \& H. A. Weaver (Tucson: Univ. Arizona Press), 223

Landolt, A. U. 1992, AJ, 104, 340

Levison, H. F. 1996, in ASP Conf. Ser. 173, Completing the Inventory of the Solar System, ed. T. W. Rettig \& J. M. Hahn (San Francisco: ASP), 173

Licandro, J., Campins, H., Hergenrother, C., \& Lara, L. M. 2003, A\&A, 398, L45

Licandro, J., Guerra, J. C., Campins, H., DiMartino, M., Lara, L. M., GilHutton, R., \& Tozzi, G. P. 2002, Earth Moon Planets, 90, 495

Licandro, J., Oliva, E., \& Di Martino, M. 2001, A\&A, 373, L29

Licandro, J., \& Pinilla-Alonso, N. 2005, ApJ, 630, L93

Licandro, J., Pinilla-Alonso, N., Pedani, M., Oliva, E., Tozzi, G. P., \& Grundi, W. M. 2006, A\&A, 445, L35

Marsden, B. G. 1974, ARA\&A, 12, 1

Marzari, F., Scholl, H., Murray, C., \& Lagerkvist, C. 2002, in Asteroids III, ed. W. F. Bottke Jr. et al. (Tucson: Univ. Arizona Press), 725

Merlin, F., Barucci, M. A., Dotto, E., de Bergh, C., \& Lo Curto, G. 2005, A\&A, 444, 977

Morbidelli, A., Levison, H. F., Tsiganis, K., \& Gomes, R. 2005, Nature, 435, 462

Oliva, E. 2000, Mem. Soc. Astron. Italiana, 71, 861

Pieters, C. M., \& Hiroi, T. 2004, in Lunar \& Planetary Science XXXV, ed. S. J. Mackwell (Houston: LPI), 1720

Pinilla, N., \& Licandro, J. 2004, in Proc. 35th COSPAR Scientific Assembly (Paris: COSPAR), 3330

Poulet, F., Cuzzi, J. N., Cruikshank, D. P., Roush, T., \& Dalle Ore, C. M. 2002, Icarus, 160,313

Rayner, J. T., Toomey, D. W., Onaka, P. M., Denault, A. J., Stahlberger, W. E., Vacca, W. D., Cushing, M. C., \& Wang, S. 2003, PASP, 115, 362

Rivkin, A. S., Howell, E. S.,Vilas, F., \& Lebofsky, L. A. 2002, in Asteroids III, ed. W. F. Bottke Jr. et al. (Tucson: Univ. Arizona Press), 235

Shkuratov, Y., Starukhina, L., Hoffman, H., \& Arnold, G. 1999, Icarus, 137, 235

Soderblom, L. A., Britt, D. T., Brown, R. H., Buratti, B. J., Kirk, R. L., Owen, T. C., \& Yelle, R. V. 2004, Icarus, 167, 100

Sunshine, J. M., et al. 2006, Science, 311, 1453

Tancredi, G., Fernández, J. A., Rickman, H., \& Licandro, J. 2000, A\&AS, 146, 73

Tholen, D. J., \& Barucci, M. A. 1989, in Asteroids II, ed. R. Binzel et al. (Tucson: Univ. Arizona Press), 298

Tokunaga, A. T., Simmons, D. A., \& Vacca, W. D. 2002, PASP, 114, 180

Zellner, B., Tholen, D. J., \& Tedesco, E. F. 1985, Icarus, 61, 355 